\title{
A randomised controlled trial on the effect of inhaled hypertonic saline on quality of life in primary ciliary dyskinesia
}

\author{
Tamara Paff ${ }^{1,2,3}$, Johannes M.A. Daniels ${ }^{1}$, Els J. Weersink ${ }^{4}$, René Lutter ${ }^{4,5}$, \\ Anton Vonk Noordegraaf ${ }^{6}$ and Eric G. Haarman ${ }^{2}$
}

Affiliations: ${ }^{1}$ Dept of Pulmonary Diseases, VU University Medical Center, Amsterdam, The Netherlands. ${ }^{2}$ Dept of Pediatric Pulmonology, VU University Medical Center, Amsterdam, The Netherlands. ${ }^{3}$ Dept of Clinical Genetics, VU University Medical Center, Amsterdam, The Netherlands. "Dept of Respiratory Medicine, Academic Medical Center, University of Amsterdam, Amsterdam, The Netherlands. ${ }^{5}$ Dept of Experimental Immunology, Academic Medical Center, University of Amsterdam, Amsterdam, The Netherlands. ${ }^{6}$ Dept of Pulmonology, Institute for Cardiovascular Research, VU University Medical Center, Amsterdam, The Netherlands.

Correspondence: Tamara Paff, VU University Medical Center, Room KTC-04-023, PO Box 7057, 1007 MB Amsterdam, The Netherlands. E-mail: t.paffavumc.nl

@ERSpublications

RCT in PCD: hypertonic saline does not improve SGRQ scores, but improves health perception http://ow.ly/uL6x306Dmzh

Cite this article as: Paff T, Daniels JMA, Weersink EJ, et al. A randomised controlled trial on the effect of inhaled hypertonic saline on quality of life in primary ciliary dyskinesia. Eur Respir J 2017; 49: 1601770 [https://doi.org/10.1183/13993003.01770-2016].

ABSTRACT Hypertonic saline inhalation lowers airway mucous viscosity. Increased cough transportability may improve quality of life (QoL) in primary ciliary dyskinesia (PCD).

In this randomised controlled trial (RCT), PCD patients received twice-daily inhalations of hypertonic (7\%) saline or isotonic (0.9\%) saline for 12 weeks, with 4 weeks washout during crossover. Primary outcome was change in QoL measured by the St George's Respiratory Questionnaire (SGRQ) total score. Secondary outcomes were SGRQ subscores, Quality of Life Questionnaire-Bronchiectasis (QoL-B) scores, lower respiratory tract infection symptoms, exacerbations, spirometry, systemic and sputum inflammatory markers, adherence, and adverse events.

There was no significant change in median (interquartile range) SGRQ total score between hypertonic saline $(-2.6(-9.0-1.5))$ and isotonic saline $(-0.3(-8.1-6.1))$ in 22 patients (age range 22-73 years) $(\mathrm{p}=0.38)$. QoL-B Health Perception scale improved with hypertonic saline $(\mathrm{p}=0.03)$. Adverse events occurred more frequently with hypertonic saline, but were mild.

12 weeks of inhaled hypertonic saline did not improve SGRQ total score in adult PCD patients in this RCT, but the sample size was small. On the secondary and more disease-specific end-point of the QoL-B, a significant improvement was observed in the Health Perception scale. This study found little evidence to support the hypothesis that hypertonic saline improves QoL in PCD patients. We advise the use of disease-specific outcome measures in future trials. 


\section{Introduction}

Mucociliary clearance (MCC) is a key part of the respiratory innate defence system, and is established by a combination of cough and synchronously beating epithelial cilia [1]. Genetic defects that cause dyskinetic or immotile cilia in primary ciliary dyskinesia (PCD) impair MCC [2]. The subsequent accumulation of mucus and susceptibility to inhaled pathogens causes recurrent airway infections, leading to chronic inflammation and airway remodelling. Daily symptoms such as cough and shortness of breath, but also pulmonary exacerbations and the progressive decline in lung function, significantly impact quality of life (QoL) in these patients [3, 4].

As there are no randomised clinical trials of PCD treatment, all recommendations are currently derived from cystic fibrosis (CF) guidelines and personal experiences [5, 6]. Despite the lack of available data, inhaled hypertonic saline is frequently prescribed to PCD patients to increase ease of sputum expectoration, thereby improving MCC. In CF, these low-cost nebulisations have been shown to dramatically prolong the time to a new exacerbation and increase health-related QoL [7-9].

The rate of MCC is known to depend on coughing, ciliary beating, volume of the airway surface layer (ASL) and rheological properties of mucus [10]. Inhaled hypertonic saline has several mechanisms of action by which it improves MCC. First, it increases ASL volume. In CF, it rehydrates the depleted ASL, but even in healthy epithelium hypertonic saline causes an osmotic shift of water, increasing the ASL volume $[8,11]$. Second, inhaled hypertonic saline lowers sputum viscosity in patients with CF and non-CF bronchiectasis, leading to a higher weight of expectorated sputum [12-14]. Third, it directly stimulates coughing [7, 15, 16]. Although results from in vitro and in vivo studies suggest that these effects are relatively short lasting, twice-daily nebulisations with hypertonic saline have shown clinical benefit in patients with $\mathrm{CF}$ and non-CF bronchiectasis $[7,8,11,17-19]$. As epithelial cilia are dysfunctional in PCD, patients are largely dependent on coughing for their mucus clearance. As sputum rheology in PCD is similar to that in CF, lowering the viscosity may improve its transportability during coughing and regular physical activity [20-22].

Despite CF and PCD sharing many clinical characteristics, they are pathophysiologically distinct and patients may therefore respond differently to various treatments [23, 24]. This is exemplified by observations in a study with DNase treatment $[25,26]$. Although not studied exclusively in PCD, but in a heterogeneous group of non-CF bronchiectasis patients, inhalations with DNase were found to be harmful in this group while being highly effective in CF. Therefore, there is an urgent need to evaluate treatment options in PCD patients.

To explore the possible effect of inhaled hypertonic saline on QoL in adults with PCD, we performed a double-blind randomised controlled crossover trial. We chose the St George's Respiratory Questionnaire (SGRQ) as our primary outcome as this is the most commonly used patient-reported outcome in non-CF bronchiectasis patients [27]. Additionally, we included the recently developed Quality of Life Questionnaire-Bronchiectasis (QoL-B), a more disease-specific patient-reported outcome, which was still under validation at the time this study was conducted [28]. We hypothesised that 12 weeks of twice-daily inhalations with hypertonic saline would improve health-related QoL in adult PCD patients compared with isotonic saline.

\section{Methods \\ Study design}

A double-blind randomised controlled crossover trial was conducted over a 28 -week period with 4 weeks washout between interventions (figure 1). Recruitment and study procedures took place between April 2014 and May 2015 at the VU University Medical Center and Academic Medical Center (Amsterdam, The Netherlands). The ethics committees of both centres approved the study and all participants gave written informed consent. The study was conducted in accordance with Good Clinical Practice guidelines and the Declaration of Helsinki, and adhered to the CONSORT guidelines (online supplementary table E1) [29]. The trial was registered at the Netherlands Trial Register (www.trialregister.nl, trial number 4674), the Dutch primary registry which is recognised and acknowledged by the World Health Organization and International Committee of Medical Journal Editors.

\section{Participants and setting}

Patients with a confirmed diagnosis of PCD, who were at least 18 years old and were in a clinically stable condition, were eligible to participate in the study. Patients were considered clinically stable when they had

Clinical trial: This study is registered at www.trialregister.nl with identifier number 4674 .

Support statement: The Dutch PCD patient support group (PCD Belangengroep) funded the study for EUR70000. Chiesi Pharmaceuticals BV donated EUR6000 for the compressors and nebulisers used in this study.

Conflict of interest: Disclosures can be found alongside this article at erj.ersjournals.com 


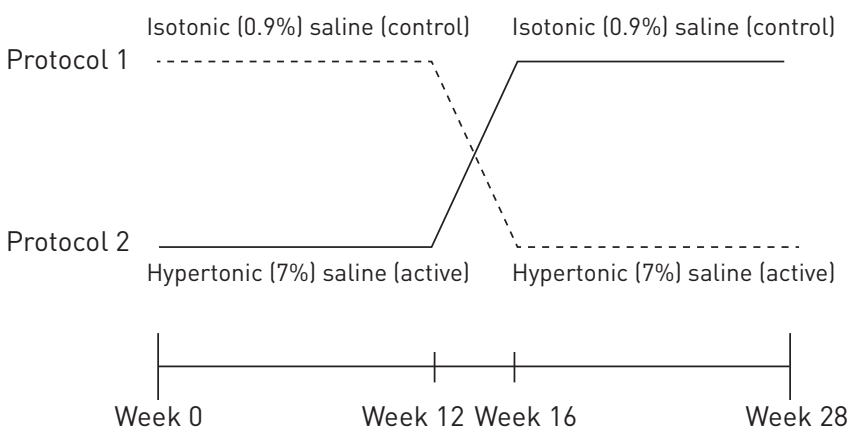

FIGURE 1 Crossover study design.

no pulmonary exacerbation in the previous 4 weeks and their forced expiratory volume in $1 \mathrm{~s}(\mathrm{FEV} 1)$ was within $10 \%$ of the best value obtained during the previous 6 months. Detailed information on diagnostic, inclusion and exclusion criteria is available in the online supplementary material. Patients were informed about the study by their treating physician or by the national patient organisation. The investigators provided detailed study information and enrolled the patients.

\section{Randomisation}

A randomisation list for the order of treatment with two equal blocks was computer-generated and provided by an external pharmacy that prepared the study medication. Allocation concealment was handled by the pharmacies of the participating centres. The investigators, participants and treating physicians were unaware of the allocation throughout the study.

\section{Interventions}

Participants received twice-daily inhalations of $5 \mathrm{~mL}$ hypertonic (7\%) saline and isotonic (0.9\%) saline, with $0.25 \mathrm{mg} \cdot \mathrm{mL}^{-1}$ quinine sulfate added to mask the taste [7]. Trial medication was packed in glass ampoules and inhaled through a mouthpiece attached to a Porta-Neb side-stream nebuliser and compressor (Philips Respironics, Amsterdam, The Netherlands). $400 \mu \mathrm{g}$ of a short-acting $\beta$-agonists bronchodilator was administered before inhalation of trial medication to prevent possible bronchoconstriction [7]. When patients did not tolerate a short-acting $\beta$-agonist, an anticholinergic was given. Standard care was maintained throughout the trial [5]. Concomitant therapies other than mucoactive treatments were approved and used at the discretion of the patient's physician.

\section{Procedures and outcomes}

After randomisation, patients underwent follow-up at weeks 6, 12, 16, 22 and 28 (figure 1). The primary end-point was change in SGRQ total score (0-100, with 100 being worst QoL) after 12 weeks of treatment [27]. Secondary end-points consisted of previously recommended treatment outcomes in PCD and established markers for monitoring lung function and sputum and systemic inflammation in chronic lung disease $[5,30]$. These included subscores of the SGRQ and the QoL-B (0-100, with 0 being worst QoL), modified lower respiratory tract infection visual analogue scale (LRTI-VAS), pulmonary exacerbations, spirometry, serum C-reactive protein, erythrocyte sedimentation rate, white blood cell count and cell differentiation, microbiological evaluation, sputum cell differentiation, sputum neutrophil elastase, interleukin- $1 \beta,-6,-8$ and -10 , tumour necrosis factor- $\alpha$, myeloperoxidase, interferon (IFN)- $\alpha$ and $-\beta$, adherence, and adverse events [28, 31]. Adherence was determined by the investigator count of all ampoules. Additional information is available in the online supplementary material.

\section{Statistical methods and sample size justification}

Statistical analysis was performed on data from the intention-to-treat population and the per-protocol population (online supplementary material). The difference in change across the treatment and control phase was tested with the paired t-test or with the Wilcoxon signed rank test if data was not normally distributed. A linear mixed model, with intervention and visit number as fixed effects, was used to analyse the pattern of change across time. Log transformation was used if data was not normally distributed.

A 4-point reduction in SGRQ total score has previously been used as the minimal clinically important difference (MCID) [32]. We set the target sample size for this study to 24 patients. This was based on a combination of sample size calculations using the reported MCID and additional subjects for possible withdrawals. A minimum sample size of 14 patients was calculated to give a power of 0.80 in order to detect an improvement of 4 points in SGRQ total score, estimating a standard deviation of 4 points, a within-patient correlation of 0.3 and a significance level of 0.05 . 


\section{Results}

\section{Study participants}

Of the 86 patients screened for eligibility, 22 patients were included in the study and underwent randomisation (see the CONSORT flow diagram in figure 2). Online supplementary table E2 shows the reasons for declining study participation. The baseline characteristics of the participants entering the study are shown in table 1 . Baseline values that were not significantly different at the start of the intervention periods were consistent with no carryover effect. Four participants discontinued the study. Three participants, of which two received hypertonic saline at the time, discontinued due to adverse events (chest tightness $(n=2)$ and nausea $(n=1)$ ), which were possibly related to the trial medication. Another participant discontinued the study following hospitalisation for treatment of a subdural haematoma, which was unrelated to the trial medication.

\section{Primary outcome}

Median (interquartile range (IQR)) change in SGRQ total score was $-2.6(-9.0-1.5)$ after 12 weeks of twice-daily hypertonic saline inhalations versus $-0.3(-8.1-6.1)$ after isotonic saline inhalations, which did not significantly differ from each other $(\mathrm{p}=0.38)$. The difference between these study periods did not reach the MCID of 4 points. Linear mixed model analysis did not show a significant effect of hypertonic saline treatment across time $(\mathrm{p}=0.57)$.

\section{Secondary outcomes}

Median changes in all health-related QoL scores are presented graphically in figures 3 and 4 for SGRQ and QoL-B, respectively. Online supplementary table E3 shows the secondary end-point data of SGRQ subscores, QoL-B scales, modified LRTI-VAS scores, spirometry, systemic inflammatory markers, sputum cell differentiation and cytokines. No statistically significant improvement in all but the QoL-B Health Perception scale and the modified LRTI-VAS score for chest pain was seen after hypertonic saline treatment compared with isotonic saline. We observed a similar number of exacerbations in both intervention phases (median (IQR) $0(0-1)$ ) and no major confounding influence of exacerbations on the

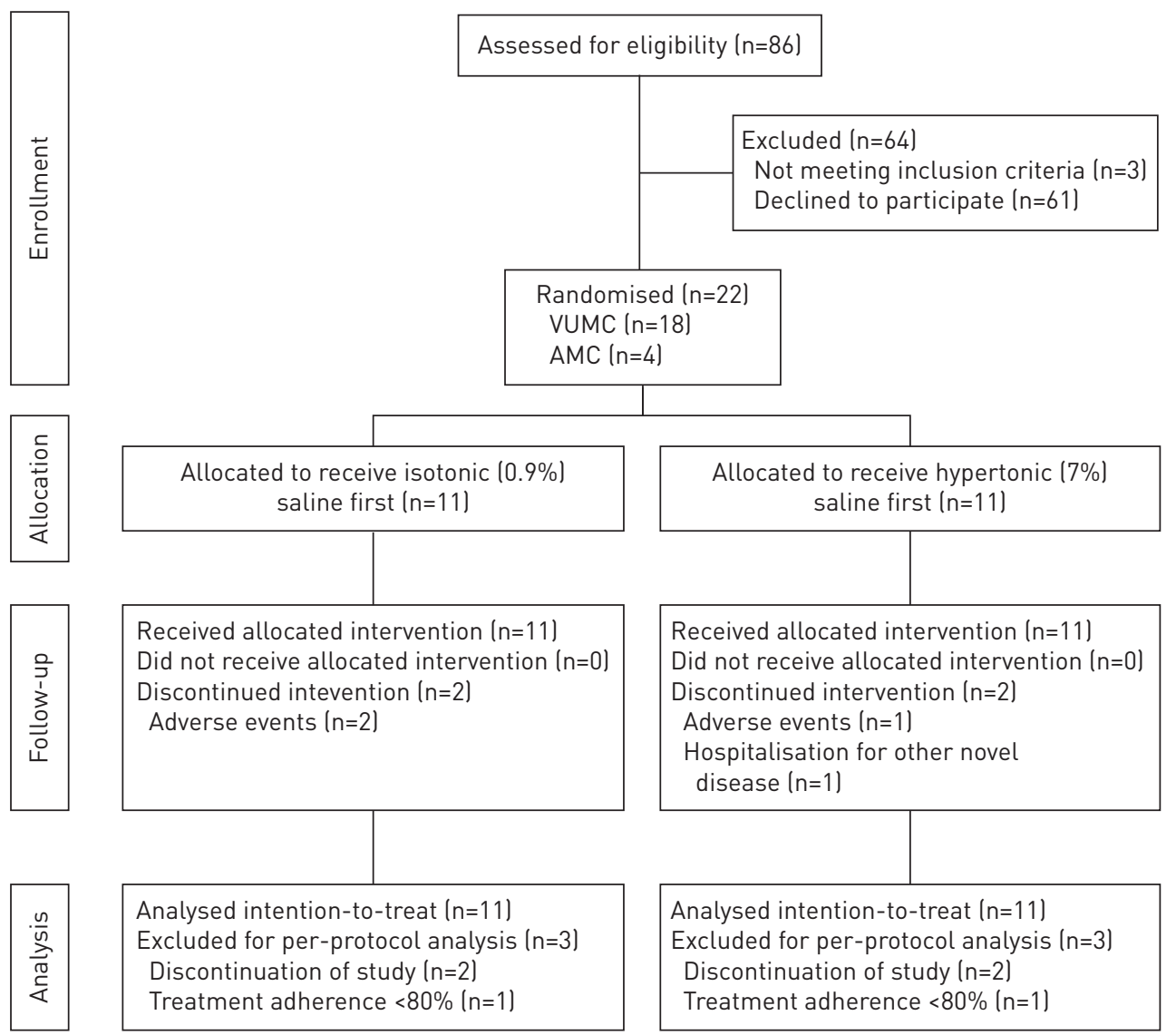

FIGURE 2 CONSORT flow chart summarising the progress of patients through the trial. VUMC: VU University Medical Center; AMC: Academic Medical Center. 
TABLE 1 Baseline characteristics

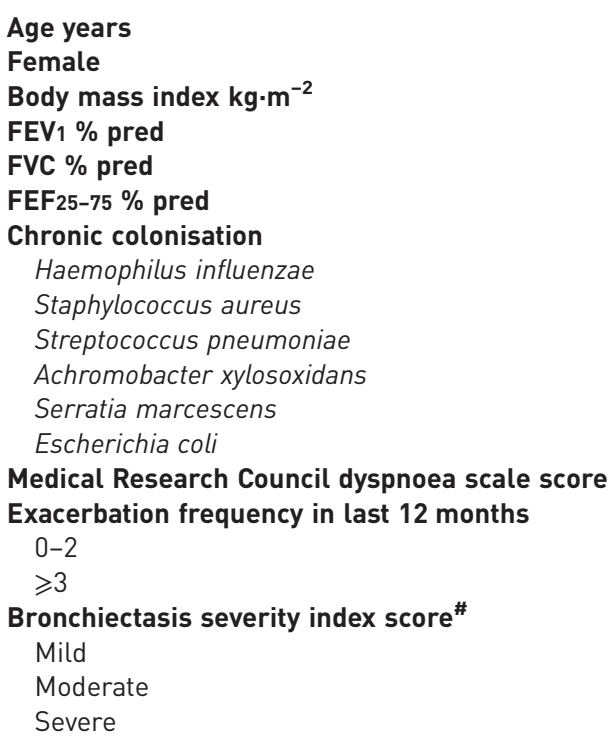

$47.6(26.9-58.1)$

$16(72.7)$

$25.8(23.6-28.7)$

75.5 (58.8-94.5)

92 (83.0-105.5)

34.5 (25.3-65.0)

10 (45.5)

7

2

1

1

Data are presented as a median (interquartile range), $\mathrm{n}(\%)$ or $\mathrm{n}$. $\mathrm{FEV} 1$ : forced expiratory volume in $1 \mathrm{~s}$; FVC: forced vital capacity; FEF25-75: forced expiratory flow at $25-75 \%$ of FVC. \#: the number of lobes affected by bronchiectasis, which was needed for calculating the bronchiectasis severity index, was based on high-resolution computed tomography images in 13 patients and on chest radiograph images in the other nine patients.

outcome parameters was observed. There were no differences in the clearance or acquisition of sputum bacteria. Online supplementary table E4 gives an overview of the responses patients gave to the open-ended question of the SGRQ in which they were asked to describe activities they feel most impaired in due to their disease.
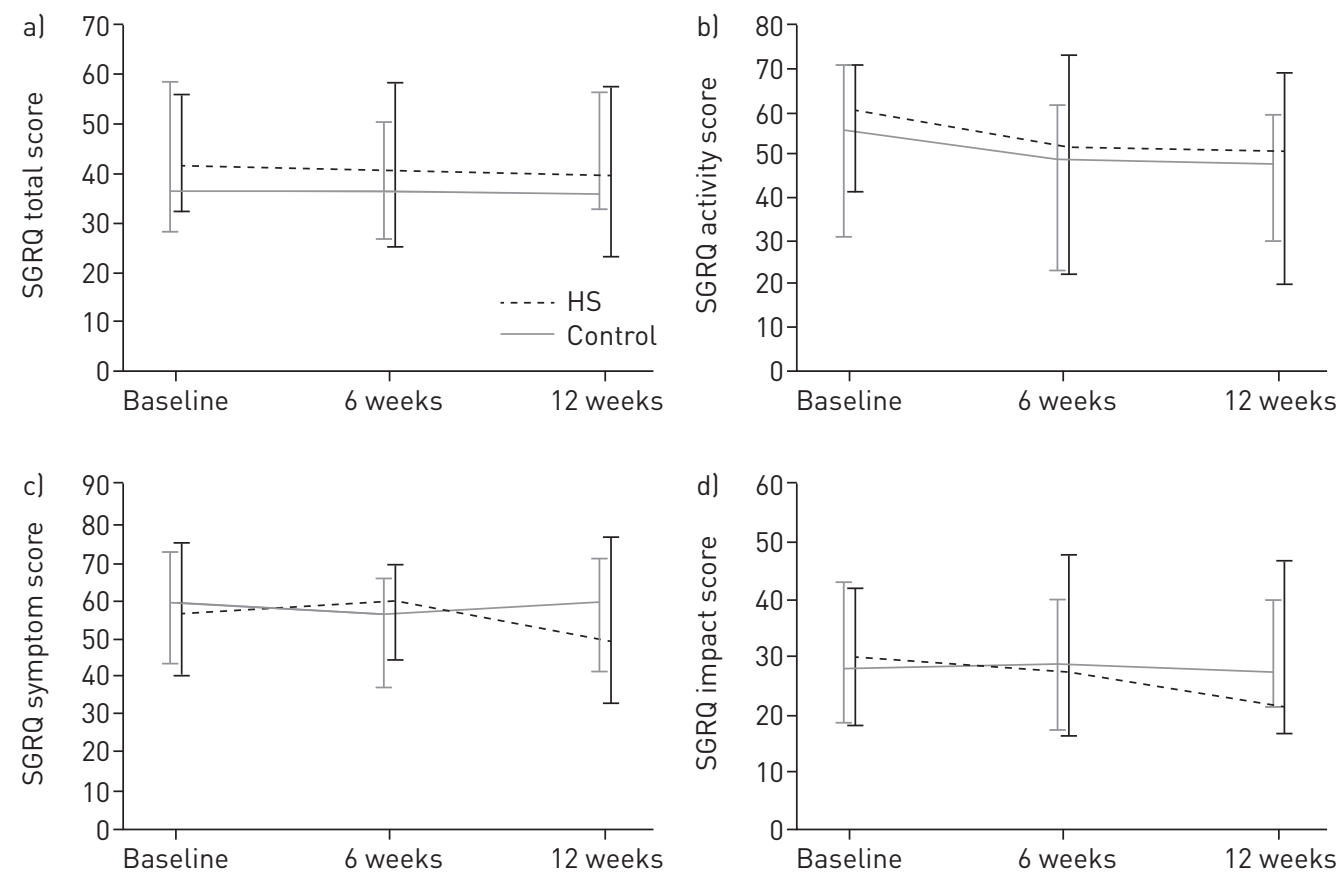

FIGURE 3 St George's Respiratory Questionnaire (SGRQ) scores with both interventions: a) total score, b) activity score, c) symptom score and d) impact score. HS: hypertonic (7\%) saline. Data are presented as median and interquartile range. 

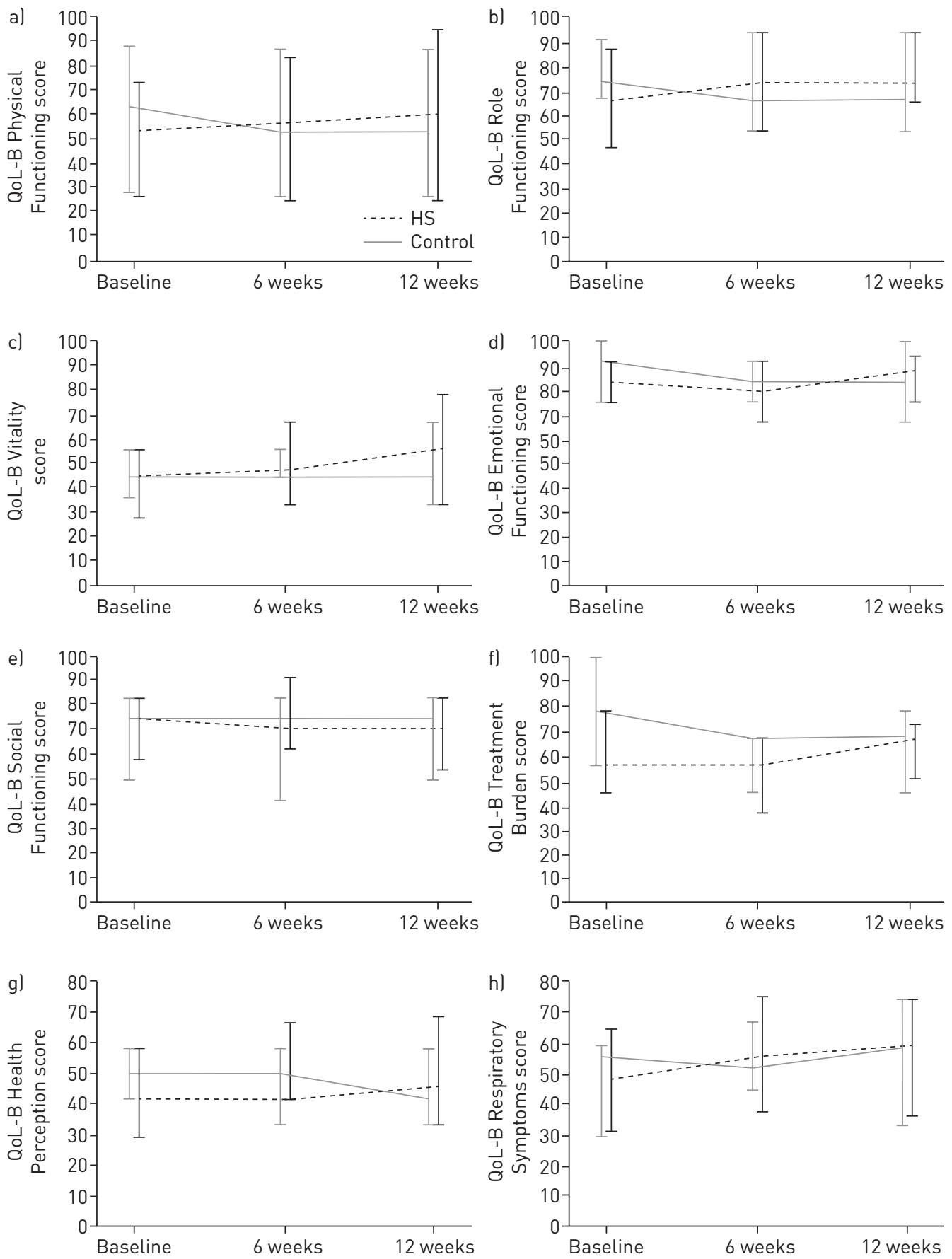

FIGURE 4 Quality of Life Questionnaire-Bronchiectasis (QoL-B) scale scores with both interventions: a) Physical Functioning score, b) Role Functioning score, c) Vitality score, d) Emotional Functioning score, el Social Functioning score, f) Treatment Burden score, g) Health Perception score and h) Respiratory Symptoms score. HS: hypertonic (7\%) saline. Data are presented as median and interquartile range.

\section{Quality of Life Questionnaire-Bronchiectasis}

The QoL-B Health Perception scale significantly improved after the hypertonic saline treatment phase compared with the control phase. Median (IQR) change was $-8.3(-16.7-8.3)$ points in the control phase versus $8.3(0.0-10.4)$ points in the hypertonic saline treatment phase $(\mathrm{p}=0.03)$, exceeding the MCID of 8 points for this item. Linear mixed model analysis did not show a significantly different change across time between both interventions. Further, we observed clinically important improvements after hypertonic saline treatment in the QoL-B Respiratory Symptoms and Vitality scales, but they did not reach statistical significance. Change in Role Functioning was borderline significantly different between the treatment and control phase $(\mathrm{p}=0.05)$, but the effects size was below the MCID. 
Modified LRTI-VAS

Modified LRTI-VAS score for chest pain improved slightly during hypertonic saline treatment phase with a median (IQR) change of $-0.4(-1.7-0.15)$ compared with the control phase with a median (IQR) change of $0.0(-0.4-0.9)(\mathrm{p}=0.03)$.

\section{Adverse events}

Adverse reactions to the study medication were seen in almost all patients, but occurred more frequently during the hypertonic saline treatment phase. Hypertonic saline most commonly caused oropharyngeal irritation, which generally subsided after a few days to weeks (table 2). Three patients were admitted to the hospital during the study for treatment of a pulmonary exacerbation, a kidney transplant rejection and a deep venous thrombosis, and for treatment of a subarachnoid haematoma. Only the latter occurred during the hypertonic saline treatment phase. None of these admissions were considered to be related to the trial medication.

Adverse events led to discontinuation of the study in three cases. Despite passing the test nebulisation, two subjects experienced symptoms which were suggestive of both bronchoconstriction and salbutamol intolerance. Another participant felt nauseated after each nebulisation with isotonic saline.

\section{Adherence}

Median (IQR) adherence to treatment, as judged by the number of returned ampoules from patients completing the study, was $93.4 \%(87.6-100 \%)$ for the hypertonic saline treatment phase and $93.0 \%(69.5-$ $98 \%)$ for the control phase $(\mathrm{p}=0.21)$. Only two participants that completed the study had $<80 \%$ compliance in both treatment phases.

\section{Per-protocol analysis}

Per-protocol analysis of patients completing the study and receiving at least a mean of $80 \%$ of trial medication in both the treatment and control phase generally had no substantial effect on the effect size and the level of significance of the outcome parameters.

Observed mean differences between the hypertonic saline and control group, observed standard deviations and within-subject variation are provided for the primary and secondary outcomes in online supplementary table E5.

\section{Discussion}

In this randomised controlled trial (RCT) we explored the effect of hypertonic saline on health-related QoL in adult PCD patients. 12 weeks of twice-daily inhalations with hypertonic saline did not significantly improve SGRQ total score in adult PCD patients. Hypertonic saline treatment did improve their health perception, as measured by the QoL-B questionnaire. In addition, a small but significant improvement in chest pain was observed, as determined by the modified LRTI-VAS. Participants generally reported low health-related QoL at baseline, similar to chronic obstructive pulmonary disease (COPD) Global Initiative for Chronic Obstructive Lung Disease grade C-D patients, but with relatively high interpatient variability depicting the clinical heterogeneity of the disease [33].

\section{TABLE 2 Adverse events possibly related to study medication}

Hypertonic $(7 \%)$ saline

Control (isotonic $(0.9 \%)$ saline)

Throat irritation/pain
Cough
Chest tightness
Increased sputum volume
Dizziness
Headache
Tiredness
Weight gain
Gastrointestinal symptoms
Haemoptysis
Tingling sensation hands/chest
Hoarseness
Bad taste in mouth

Data are presented as $\mathrm{n}$. 
There are several possible explanations for the lack of a statistically significant difference between SGRQ scores of patients treated with hypertonic saline and isotonic saline. First, the observed difference in change of SGRQ scores after hypertonic saline treatment was smaller than the MCID, and the intersubject variability was larger than estimated. Consequently, the sample size was too small to detect these changes. In order to make informed sample size calculations in future larger trials we added detailed information on the observed differences and the variability (online supplementary table E5). Second, hypertonic saline may not improve QoL in PCD patients as it does in CF. In CF, the ASL is dehydrated due to depletion of salt. In PCD and other non-CF bronchiectasis, where there is no problem with CF transmembrane conductance regulator function causing electrolyte shifts but mucous is viscous due to inflammatory cells and cell debris, hydration may not have the same effect [20]. However, when sputum from CF and non-CF bronchiectasis patients was incubated with saline and placed on bovine trachea, a comparable dose-dependent improvement in transportability was observed [34]. In contrast to this animal model and other causes of bronchiectasis, epithelial cilia in PCD are dysfunctional and a lower viscosity will not lead to improved ciliary clearance in these patients. However, hypertonic saline was also shown to improve cough clearability [14]. Coughing, the primary clearance mechanism in PCD, was shown to remove a similar fraction of inhaled particles in PCD patients as in healthy nonsmoking subjects, but it does take more time to achieve [22]. Third, SGRQ total score may not have been sensitive enough to detect a clinical benefit in PCD patients. The scores for QoL-B Health Perception, Respiratory Symptoms and Vitality did show improvements that exceeded the MCID after hypertonic saline treatment, although only the first reached statistical significance. Naturally, these results need to be interpreted with caution as we did not correct for multiple testing in this first explorative study. In contrast to the SGRQ, the QoL-B is specifically designed to assess respiratory symptoms in bronchiectasis patients instead of COPD patients. It includes important symptoms that are very common in PCD, e.g. "the feeling of a full chest", which are not addressed in the SGRQ. Furthermore, the QoL-B was developed with US Food and Drug Administration patient-reported outcome guidance, has fewer items, and has a shorter and less variable recall. This may suggest that it is important to use a disease-specific patient-reported outcome as primary outcome in RCTs to fully address the issues PCD patients face. A questionnaire encompassing all PCD-specific symptoms (PCD-QoL), including items on the ear, nose and throat (ENT), was recently developed as part of the international FP7 BESTCILIA programme [35]. Hypertonic saline treatment is not expected to relieve ENT symptoms when delivered by a mouthpiece, but the use of a face mask may be beneficial. This also holds true for interventions with a more systemic effect [36].

In contrast to this study, NICOLSON et al. [17] showed improved SGRQ scores and spirometric values in non-CF bronchiectasis patients after 12 months of treatment. However, these improvements were observed in both the treatment and control group, suggesting that hypertonic saline and isotonic saline may have similar beneficial effects or that patients benefited from participation in the trial, including the frequent check-ups. There is some evidence in COPD that isotonic saline could also have a positive clinical effect by humidifying the airway surface liquid [37]. However, the effect of nebulised saline is strongly dose dependent and isotonic saline was not observed to be of clinical benefit in the large CF trials [38-40]. In our study we also did not see any improvement in SGRQ total score following inhalations with isotonic saline, but sample size was small. In contrast to the present study and Nicolson et al. [17], KeLletT et al. [18] did observe superiority of hypertonic saline over isotonic saline in QoL and exacerbation rate after only 3 months of treatment. However, with the relatively low exacerbation rate that we observed one would need a longer study duration to address this. Additionally, our study is the only one using an appropriate taste-masking agent, as was also done in the CF trials $[7,8]$. The lack of masking the distinct taste of hypertonic saline may have caused inadequate blinding. Further, both the NiColson et al. [17] and KelLEtT et al. [18] studies were conducted in non-CF bronchiectasis patients, whereas this study was done in PCD patients only. Non-CF bronchiectasis comprises a heterogeneous group of patients with bronchiectasis of various origins, including post-infectious bronchiectasis, immune deficiencies, autoimmune diseases, PCD and a large group of unknown aetiology. Bronchiectasis of different aetiology may respond differently to treatment. In addition, not all our patients had bronchiectasis.

The absence of a clinically important effect of hypertonic saline treatment on spirometry that we observed is in line with the Nicolson et al. [17] study, but not with the Kellett et al. [18] study. A reason for this could be the relatively good lung function of patients in the current and Nicolson et al. [17] study compared with the KelletT et al. [18] study (FEV1 \% pred 75-85\% versus 66\%, respectively) [41].

The present study is the first to report longitudinal data on airway inflammation in PCD patients. Similar to previous cross-sectional studies in PCD, we found severe neutrophil-dominated inflammation and high levels of pro-inflammatory cytokines [20,42-44]. We did not observe any change in sputum inflammatory markers after hypertonic saline treatment in our cohort, which is in line with data previously obtained in CF patients [7]. Our results extend these findings by also showing remarkably high levels of IFN- $\alpha$ and $-\beta$. 
While the role of these type 1 IFNs in antiviral innate immunity is well established, evidence about their possible ambivalent role in bacterial infections has been accumulating in the past few years [45].

We observed a relatively low participation rate of $26.5 \%$ in PCD patients that were eligible to participate in this study. Interestingly, the two main reasons for declining participation were either 1) too time consuming (hospital too far away or too much time spent on study medication and study visits) or 2) that they were not willing to stop and washout their hypertonic saline treatment that they were currently using. The first reason could be partially specifically related to the Netherlands, as inhabitants are not used to travelling long distances to a hospital and study procedures were only performed in the two academic hospitals in Amsterdam. We are not exactly sure how to avoid the second issue in future trials, although the prescribing rate of hypertonic saline in PCD patients may be lower in other countries.

In summary, this double-blind RCT demonstrated no significant effect of hypertonic saline in adult PCD patients on the primary outcome, i.e. SGRQ total score. We did observe a significant improvement of the Health Perception scale of the more disease-specific QoL-B and a small but significant improvement in the feeling of chest pain, measured by the modified LRTI-VAS. Based on these results, we advise that a disease-specific patient-reported outcome is used in future studies.

\section{Acknowledgements}

We thank the patients who volunteered to participate in this study; the pharmacy of the University Medical Center St Radboud (Nijmegen, The Netherlands) for manufacturing the trial medication; Tamara Dekker and Barbara Smids-Dierdorp (Dept of Experimental Immunology Academic Medical Center, Amsterdam, The Netherlands) and Pieter Hiemstra and Bram van der Linden (Dept of Pulmonology, Leiden University Medical Center, Leiden, The Netherlands) for their work on sputum cell counts and inflammatory parameters; and Birgit Witte (Dept of Epidemiology and Biostatistics, VU University Medical Center, Amsterdam, The Netherlands) for her work on the statistical analysis.

Author contributions: All authors approved this version to be published and agree to be accountable for all aspects of the work. T. Paff made substantial contributions to the acquisition, analysis and interpretation of the data, and drafting of the manuscript. J.M.A. Daniels and E.G. Haarman made substantial contributions to the design of the study, patient recruitment and interpretation of the data, and they critically revised the manuscript for important intellectual content. E.J. Weersink and A. Vonk Noordegraaf made substantial contributions to patient recruitment, interpretation of the data and revision of the manuscript for intellectual content. R. Lutter substantially contributed to acquisition, analysis and interpretation of the data, and revision of the manuscript.

\section{References}

1 Knowles M, Boucher R. Mucus clearance as a primary innate defense mechanism for mammalian airways. J Clin Invest 2002; 109: 571-577.

2 Knowles MR, Daniels LA, Davis SD, et al. Primary ciliary dyskinesia. Recent advances in diagnostics, genetics, and characterization of clinical disease. Am J Respir Crit Care Med 2013; 188: 913-922.

3 Pifferi M, Bush A, Di Cicco M, et al. Health-related quality of life and unmet needs in patients with primary ciliary dyskinesia. Eur Respir J 2010; 35: 787-794

4 McManus IC, Mitchison HM, Chung EMK, et al. Primary ciliary dyskinesia (Siewert's/Kartagener's Syndrome): respiratory symptoms and psycho-social impact. BMC Pulm Med 2003; 3: 4.

5 Barbato A, Frischer T, Kuehni CE, et al. Primary ciliary dyskinesia: a consensus statement on diagnostic and treatment approaches in children. Eur Respir J 2009; 34: 1264-1276.

6 Shapiro AJ, Zariwala MA, Ferkol T, et al. Diagnosis, monitoring, and treatment of primary ciliary dyskinesia: PCD foundation consensus recommendations based on state of the art review. Pediatr Pulmonol 2015; 51: 115-132.

7 Elkins M, Robinson M, Rose B, et al. A controlled trial of long-term hypertonic saline in patients with cystic fibrosis. N Engl Med J 2006; 354: 229-240.

8 Donaldson SH, Bennett WD, Zeman KL, et al. Mucus clearance and lung function in cystic fibrosis with hypertonic saline. N Engl J Med 2006; 354: 241-250.

9 Wark P, Mcdonald V. Nebulised hypertonic saline for cystic fibrosis. Cochrane Database Syst Rev 2009; 2: CD001506.

10 Wanner A, Salathe M, Riordan TGO. Mucociliary clearance in the airways. Am J Respir Crit Care Med 1996; 154: 1868-1902.

11 Tarran R, Grubb BR, Parsons D, et al. The CF salt controversy: in vivo observations and therapeutic approaches. Mol Cell 2001; 8: 149-158.

12 Kellett F, Redfern J, Niven R. Evaluation of nebulised hypertonic saline (7\%) as an adjunct to physiotherapy in patients with bronchiectasis. Respir Res 2005; 99: 27-31.

13 Dasgupta B. Combined effects of hypertonic saline and rhDNase on cystic fibrosis sputum in-vitro. Pediatr Pulmonol 1995; 20: A201-A236.

14 King M, Dasgupta B, Tomkiewicz RP, et al. Rheology of cystic fibrosis sputum after in vitro treatment with hypertonic saline alone and in combination with recombinant human deoxyribonuclease I. Am J Respir Crit Care Med 1997; 156: 173-177.

15 Koskela HO, Kontra KM, Purokivi MK, et al. Interpretation of cough provoked by airway challenges. Chest 2005; 128: 3329-3335.

16 Robinson M, Daviskas E, Eberl S, et al. The effect of inhaled mannitol on bronchial mucus clearance in cystic fibrosis patients: a pilot study. Eur Respir J 1999; 14: 678-685.

17 Nicolson CHH, Stirling RG, Borg BM, et al. The long term effect of inhaled hypertonic saline $6 \%$ in non-cystic fibrosis bronchiectasis. Respir Med 2012; 106: 661-667. 
18 Kellett F, Robert NM. Nebulised 7\% hypertonic saline improves lung function and quality of life in bronchiectasis. Respir Med 2011; 105: 1831-1835.

19 Bennett WD, Wu J, Fuller F, et al. Duration of action of hypertonic saline on mucociliary clearance in the normal lung. J Appl Physiol 2015; 118: 1483-1490.

20 Bush A, Payne D, Pike S, et al. Mucus properties in children with primary ciliary dyskinesia: comparison with cystic fibrosis. Chest 2006; 129: 118-123.

21 Phillips GE, Thomas S, Heather S, et al. Airway response of children with primary ciliary dyskinesia to exercise and $\beta_{2}$-agonist challenge. Eur Respir J 1998; 11: 1389-1391.

22 Möller W, Häussinger K, Ziegler-Heitbrock L, et al. Mucociliary and long-term particle clearance in airways of patients with immotile cilia. Respir Res 2006; 7: 10.

23 Lucas J, Caroll M. Primary ciliary dyskinesia and cystic fibrosis: different diseases require different treatment. Chest 2014; 145: 674-676.

24 Cohen-Cymberknoh M, Simanovsky N, Hiller N, et al. Differences in disease expression between primary ciliary dyskinesia and cystic fibrosis with and without pancreatic insufficiency. Chest 2014; 145: 738-744.

25 Wills PJ, Wodehouse T, Corkery K, et al. Short-term recombinant human DNase in bronchiectasis: effect on clinical state and in vitro sputum transportability. Am J Respir Crit Care Med 1996; 154: 413-417.

26 O'Donnell AE, Barker AF, Ilowite JS, et al. Treatment of idiopathic bronchiectasis with aerosolized recombinant human DNase I. Chest 1998; 113: 1329-1334.

27 Wilson CB, Jones PW, O'Leary CJ, et al. Validation of the St. George's Respiratory Questionnaire in bronchiectasis. Am J Respir Crit Care Med 1997; 156: 536-541.

28 Quittner AL, O'Donnell AE, Salathe MA, et al. Quality of Life Questionnaire-Bronchiectasis: final psychometric analyses and determination of minimal important difference scores. Thorax 2015; 70: 12-20.

29 Schulz KF, Altman DG, Moher D, et al. CONSORT 2010 Statement: updated guidelines for reporting parallel group randomised trials. BMC Med 2010; 8: 18 .

30 Sagel SD, Davis SD, Campisi P, et al. Update of respiratory tract disease in children with primary ciliary dyskinesia. Proc Am Thorac Soc 2011; 8: 438-443.

31 Altenburg J, de Graaff C, Stienstra Y, et al. Effect of azithromycin maintenance treatment on infectious exacerbations among patients with non-cystic fibrosis bronchiectasis. The BAT randomized controlled trial. JAMA 2013; 309: 1251-1259.

32 Jones PW. St. George's Respiratory Questionnaire: MCID. COPD J Chronic Obstr Pulm Dis 2005; 2: 75-79.

33 Han MK, Muellerova H, Curran-Everett D, et al. GOLD 2011 disease severity classification in COPDGene: a prospective cohort study. Lancet Respir Med 2014; 1: 43-50.

34 Wills PJ, Hall RL, Chan W, et al. Sodium chloride increases the ciliary transportability of cystic fibrosis and bronchiectasis sputum on the mucus-depleted bovine trachea. J Clin Invest 1997; 99: 9-13.

35 Lucas JS, Behan L, Dunn Galvin A, et al. A quality-of-life measure for adults with primary ciliary dyskinesia: QOL-PCD. Eur Respir J 2015; 46: 375-383.

36 Kobbernagel HE, Buchvald FF, Haarman EG, et al. Study protocol, rationale and recruitment in a European multi-centre randomized controlled trial to determine the efficacy and safety of azithromycin maintenance therapy for 6 months in primary ciliary dyskinesia. BMC Pulm Med 2016; 16: 104.

37 Khan SY, O'Driscoll BR. Is nebulized saline a placebo in COPD? BMC Pulm Med 2004; 4: 312-317.

38 Daviskas E, Robinson M, Anderson SD, et al. Osmotic stimuli increase clearance of mucus in patients with mucociliary dysfunction. J Aerosol Med 2002; 15: 331-341.

39 Robinson M, Regnis JA, Bailey DL, et al. Effect of hypertonic saline, amiloride, and cough on mucociliary clearance in patients with cystic fibrosis. Am J Respir Crit Care Med 1996; 153: 1503-1509.

40 Robinson M, Hemming AL, Regnis JA, et al. Effect of increasing doses of hypertonic saline on mucociliary clearance in patients with cystic fibrosis. Thorax 1997; 52: 900-903.

41 Hart A, Sugumar K, Milan S, et al. Inhaled hyperosmolar agents for bronchiectasis. Cochrane Database Syst Rev 2014; 5: CD002996.

42 Zihlif N, Paraskakis E, Lex C, et al. Correlation between cough frequency and airway inflammation in children with primary ciliary dyskinesia. Pediatr Pulmonol 2005; 39: 551-557.

43 Kupfer $\mathrm{O}$, Wagner B, Knowles $\mathrm{M}$, et al. Airway inflammation in a large cohort of children with primary ciliary dyskinesia. Am J Respir Crit Care Med 2013; 187: A2086.

44 Hilliard TN, Regamey N, Shute JK, et al. Airway remodelling in children with cystic fibrosis. Thorax 2007; 62: 1074-1080

45 Trinchieri G. Type I interferon: friend or foe? J Exp Med 2010; 207: 2053-2063. 